The Design of Electric Vehicle Charging Network 


\title{
THE DESIGN OF ELECTRIC VEHICLE CHARGING NETWORK
}

\author{
BY
}

XIAOZHOU ZHANG, B.Eng.

\author{
A THESIS \\ Submitted to the School of Computational Science and Engineering \\ AND THE SCHOOL OF GRADUATE STUDIES \\ OF MCMASTER UNIVERSITY \\ IN PARTIAL FULFILMENT OF THE REQUIREMENTS \\ FOR THE DEGREE OF \\ MASTER OF SCIENCE
}

(c) Copyright by Xiaozhou Zhang, September 2015

All Rights Reserved 
Master of Science (2015)

(Computational Science and Engineering)
McMaster University

Hamilton, Ontario, Canada

TITLE: The Design of Electric Vehicle Charging Network

AUTHOR:

Xiaozhou Zhang

B.Eng., (Geographic Information System)

China University of Geosciences, Wuhan, China

SUPERVISOR: $\quad$ Dr. Kai Huang

NUMBER OF PAGES: viii, 51 
To my beloved parents and sister 


\section{Abstract}

The promotion of Electric Vehicles (EV) has become a key measure of the governments to reduce greenhouse gas emissions. However, range anxiety is a big barrier for drivers to choose EVs over traditional vehicles. Installing more charging stations in appropriate locations can relieve EV drivers' range anxiety. To help decide the location and number of public charging stations, we propose two optimization models for two different charging modes - fast and slow charging, which aim at minimizing the total cost while satisfying certain spatial coverage goals. Instead of using discrete points we employ network and polygons to represent charging demands. Importantly, we resolve the partial coverage problem (PCP) by segmenting the geometric objects into smaller ones using Geographic Information System (GIS) functions. We compare the geometric segmentation method (GS) and the complementary partial coverage method (CP) developed by Murray (2005) to solve the PCP. After applying the models to Greater Toronto and Hamilton Area (GTHA) and to Downtown Toronto, we show that that the proposed models are practical and effective in determining the locations and number of required charging stations. Moreover, comparison of the two

methods shows that GS can fully eliminate PCP and provide much more accurate result than $\mathrm{CP}$. 


\section{Acknowledgements}

I feel deeply grateful to my supervisor, Prof. Kai Huang, for his guidance and brilliant advice on my study and research. His broad knowledge and advice helped me all along from the formulation of research ideas to the writing of this thesis.

I also would like to express my gratitude to Prof. Pavlos Kanaroglou, who introduced me to the world of transportation models and offered great advice on my thesis.

Besides, I also would like to thank my thesis examination committee member, Prof. Manish Verma for taking the time to review my thesis.

My sincere thanks to Dr. Ron Dalumpines, for his generous help on the experiment data of this thesis and my classmate Fan E, for her constructive advice on the research and help on my research presentation.

Many thanks to my fellow classmates Miaodi Sun, James Arias, Ali Ghassemi, Sergei Melkoumian for the time studying together and fun time we spent together, which brought me a lot of motivation and inspiration.

Last but not the least, I would like to thank my parents and sister for their endless support through my study and life. 


\section{Contents}

Abstract $\quad$ iv

Acknowledgements $\quad$ v

1 Introduction 1

2 Literature Review 5

3 Partial Coverage Problem $\quad 10$

4 Fast Charging Stations $\quad 18$

4.1 Framework . . . . . . . . . . . . . . . . . . . . 19

4.2 Optimization Model . . . . . . . . . . . . . . . . . 21

5 Slow Charging Stations $\quad 24$

5.1 Framework ....................... 25

5.2 Optimization Model . . . . . . . . . . . . . . . . . 27

$6 \quad$ Numerical Experiments $\quad 29$

6.1 Fast Charging Stations . . . . . . . . . . . . . . . . . 29

6.2 Slow Charging Stations . . . . . . . . . . . . . . 34 
6.3 Estimating Demand for Each Station . . . . . . . . . . . .

7 Conclusion

A Appendix

46 


\section{List of Figures}

3.1 Partial coverage problem for lines . . . . . . . . . . . . . . 11

3.2 Partial coverage problem for polygons . . . . . . . . . . . . 12

3.3 Redundant non-complementary partial coverage . . . . . . . . . 13

4.4 Splitting partially covered link . . . . . . . . . . . . . 20

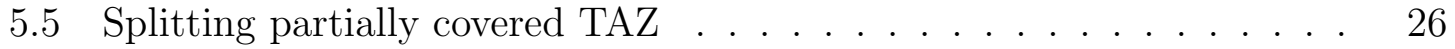

6.6 Coverage gap . . . . . . . . . . . . . . . . . . . . . . . . . 33

6.7 Comparison of visualized results $(\mathrm{R}=15 \mathrm{~km}$, alpha $=90 \%) \ldots \ldots 34$

6.8 Comparison of visualized results $(\mathrm{R}=500 \mathrm{~m}$, alpha $=90 \%) \ldots 37$

6.9 Divide fast charging demand . . . . . . . . . . . . . . 38

6.10 Thiessen polygons . . . . . . . . . . . . . . . . . . . 39

6.11 Thiessen polygons splitting shared demand . . . . . . . . . . . 41

6.12 Divide slow charging demand . . . . . . . . . . . . . 43

A.13 Visualized result for SC-CP $(\mathrm{R}=500 \mathrm{~m}$, alpha $=90 \%$, beta $=1$, omega $=33 \%$, theta $=3) \ldots \ldots \ldots \ldots \ldots$ 


\section{Chapter 1}

\section{Introduction}

Many countries around the world are drawing up plans to electrify their transportation systems in order to reduce greenhouse gas emission and to improve air quality in urban areas. The core of such plans is to promote the adoption of Electric Vehicles (EVs). However, range anxiety is one of the primary barriers for drivers to choose EVs over traditional Internal Combustion Engine (ICE) vehicles (Eberle and von Helmolt, 2010). Installing more EV charging stations is one of the strategies that can reduce range anxiety. This leads to a facility location problem: how many charging stations do we need and where are the best locations to install those charging stations? The answer of this problem depends on many factors, including the driving ranges of EVs and the cost of charging stations.

The driving range of EVs can vary greatly by model and manufacturer. Currently, the longest EV driving range is $424 \mathrm{~km}$ (2014 Tesla Model S) while the shortest range is $60 \mathrm{~km}$ (2013 Scion iQ EV). Most EVs have ranges between $100 \mathrm{~km}$ and $160 \mathrm{~km}$ (U.S. Department of Energy, 2014).

EVs are charged through Electric Vehicle Supply Equipment (EVSE). According 
M.Sc. Thesis - Xiaozhou Zhang McMaster - Computational Science and Engineering

to Community Energy Association (2013), there are three levels of EVSEs. Level 1 EVSE, with a cost less than $\$ 1000$, typically takes $10-20$ hours to charge. The long charging time makes Level 1 chargers suitable only for home usage. Level 2 can be used for both commercial and home charging purposes. EVs will take 4-8 hours to reach a full charge. Commercial Level 2 charging equipment costs between $\$ 3,500$ and $\$ 6,000$ for a single cord station while residential Level 2 charging equipment is much cheaper with cost around $\$ 1,000$. Level 3 EVSE, also called fast charger, provides the fastest way of charging EVs and can achieve 50\% charge in 10 to 15 minutes. It's also the most expensive EVSE with its cost ranging between $\$ 60,000$ and $\$ 100,000$.

Home, work and public charging are three common EV charging scenarios (National Renewable Energy Laboratory, 2014). Home charging is the dominant charging scenario. At least $70 \%$ of the electricity that EVs use is charged at home (National Renewable Energy Laboratory, 2014). Work charging happens at workplace where people park their EVs during working hours. Public charging usually occurs at public places such as shopping malls, hotels, restaurants, or public parking lots. Due to different charging time required for different levels of EVSEs, Level 1 and Level 2 EVSEs are suitable for home and work charging. Level 2 and Level 3 are suitable for public charging.

In this paper we focus on the design of a network of public charging stations. We propose to locate the Level $2 \& 3$ charging stations based on different standards of ranges. For Level 2 charging stations, because it usually takes hours to fully charge an $\mathrm{EV}$, the EVs are often charged at the parking spaces while the drivers are conducting some other activities, e.g., shopping or dining. Therefore, the drivers will look for charging stations within walking distance of the activity. Level 3 EVSE charges much 
M.Sc. Thesis - Xiaozhou Zhang McMaster - Computational Science and Engineering

faster, requiring about 30 minutes for a complete charge. Thus, it is appropriate for mid-trip charging where the drivers usually conduct long distance driving and expect to charge the EVs fast (Community Energy Association, 2013). In this scenario, the drivers will look for charging stations within driving distance before the battery is depleted.

Most traditional facility location models assume that the demands come from discrete points, which are single-dimensional (Miller, 1996; Chen et al., 2013; Frade et al., 2011; Xi et al., 2013). This approach is problematic and inaccurate for the problem in this paper. It can cause error when measuring the distance between the demand and the service facility, thus affecting the result of the facility locations (Miller, 1996). Moreover, models using point representation suffer from the partial coverage problem (PCP) and Modifiable Areal Unit Problem (MAUP), which will be introduced in Chapter 3. To ensure better accuracy, we assume that the sources of demand are multi-dimensional geometric objects, which are a better representation of reality. We model the public Level 2 charging demand using Traffic Analysis Zones (TAZ) (polygons) and the public Level 3 charging demand using links of the traffic network (lines).

To identify the optimal locations for EV charging stations, we propose a method which considers both Level 2 and Level 3 charging stations using the same framework. Compared to previous similar studies on the locations of EV charging stations, this paper has three major innovations (Frade et al., 2011; Liu, 2012; Lee et al., 2014). First, this model focuses on addressing range anxiety, making sure the charging stations are accessible to the largest possible number of EVs within allowed distances. Moreover, we discuss different definitions of range anxiety. Second, we use 
$\underline{\text { M.Sc. Thesis - Xiaozhou Zhang McMaster - Computational Science and Engineering }}$

network/polygon segmentation techniques to avoid partial coverage, which can cause models to be inaccurate. Third, the model can be applied to Level 2 and Level 3 charging stations under the same framework, offering a more comprehensive solution strategy than existing models.

This model may be used by city planners to plan the EV public charging infrastructures, by businesses to estimate how many charging stations they need to install to fulfill their customers' needs or by utility companies to estimate the impact of the charging loads on the grid network.

This paper is organized as follows. Section 2 reviews the literature of charging network design. Section 3 discusses the PCP and the classical method to address it using complimentary partial coverage. Section 4 describes the framework and mathematical formulation for fast charging stations (Level 3). Section 5 describes the framework and mathematical formulation for slower charging stations (Level 2). Finally, in Section 6, we apply the proposed model for Level 3 and Level 2 to the Greater Toronto and Hamilton Area (GTHA) and Downtown Toronto respectively, and conduct numerical studies to demonstrate the effectiveness of the proposed method. 


\section{Chapter 2}

\section{Literature Review}

Numerous efforts have been made to tackle the EV charging station location problem. In the remainder of this paper, we refer to Level 3 as fast charging and to Level 2 as slow charging.

A large number of models have been developed for fast charging stations (Ge et al., 2011; Chen et al., 2014; Hanabusa and Horiguchi, 2011; Lee et al., 2014; Lam et al., 2014). A fast charging station serves mid-trip charging needs, so that the charging demand is usually based on the number of EVs on the road and is closely related to EV users' travelling behavior. Traffic assignment is a commonly used tool for modeling the EV drivers' behavior (Lam and Lo, 2004). Hanabusa and Horiguchi (2011) apply the stochastic user equilibrium method to estimate the traffic flow on the road network. The goal of their model is to minimize the system's total travel time and equalize the charging load among charging stations using entropy maximization. Their model focuses on the impact of charging stations on EV driver's route choice but doesn't address the accessibility of charging stations to EVs. Chen et al. (2014) also utilize user equilibrium traffic assignment method to model the traffic flows, however, no 
M.Sc. Thesis - Xiaozhou Zhang McMaster - Computational Science and Engineering

facility location optimization model is developed to determine the optimal locations of charging stations. Lee et al. (2014)'s stochastic model incorporates the traffic assignment method endogenously and minimizes the total travel time and trip failure penalty. With the exception of Lee et al. (2014)'s model, most of the models for Level 3 do not address the range anxiety issue by ignoring the driving range of EVs. But Lee et al. (2014)'s model does not guarantee that EVs are able to reach a charging station within a given distance. Lam et al. (2014) formulate the problem using a network flow model of which the objective is to make sure every node on the network has at least one adjacent charging station and the charging station sub-graph remains a connected graph with the lowest cost. They use four different methods to solve the problem, including a meta-heuristic algorithm Chemical Reaction Optimization. But their model fails to consider the accumulation of demand on each charging station when determining the station's charging load. Also, their model suffers from serious scalability issues.

Some studies are dedicated to slow charging stations (Frade et al., 2011; Xi et al., 2013; Chen et al., 2013). These models generally use regression methods to estimate charging demand of each area in the city. Frade et al. (2011) estimate the daytime and nighttime charging demand in each traffic zone based on employment and residence data respectively and then use an optimization model to maximize the total coverage with a given number of charging stations. Xi et al. (2013) estimate the charging need for each TAZ and the model aims at maximizing the usage of the chargers. Chen et al. (2013) also use regression analysis based on travel survey data to estimate charging demand in each TAZ and develop an optimization model to minimize the total access cost of EVs to their nearest charging stations. In Frade 
M.Sc. Thesis - Xiaozhou Zhang McMaster - Computational Science and Engineering

et al. (2011) and Chen et al. (2013)'s models, when the distance between a TAZ and a charging station is estimated, the centroid of a TAZ is used to approximate the zone. This approach is problematic and inaccurate: It does not distinguish whether an area is partially covered or fully covered. This problem can be resolved by the segmentation technique introduced in Sections 3 and 4 of this paper.

Comparing the models for slow charging stations with those for fast charging stations, it is clear that models for fast charging station are based on the traffic network (lines) while models for slow charging station are based on zones (polygons). Importantly, both slow and fast charging stations should be built to tackle the range anxiety problem. Indeed, fast charging is indispensable for resolving range anxiety, since long distance drivers need fast charging solutions. Nevertheless, fast charging costs more and sets higher requirement on the capacity of the electric grid. As a result, slow charging remains the dominant charging mode at present.

Most existing models only consider either slow or fast charging stations. To the best of our knowledge, there is only one paper discussing multiple charging modes. Liu (2012) uses an ad hoc method to estimate the number of Level 1\&2 charging posts in each residence community and parking lot based on economic and industrial data, and decides the locations of Level 3 fast charging stations according to the locations of gas stations. This ad hoc method requires less computation and is easy to implement, but the result is less reliable than those obtained through optimization models.

Table 2.1 is a summary of the models mentioned above. As noted in the second column, most fast charging models use traffic assignment to estimate charging demand while slow charging models estimate the demand using regression method. In the third 
M.Sc. Thesis - Xiaozhou Zhang McMaster - Computational Science and Engineering

column, with the exception of Chen et al. (2014) and Liu (2012), all the models use optimization to decide the locations of charging stations. In the fourth column, typical objectives among the models include minimizing travel time and cost, and maximizing coverage. As we can see from the fifth column, slow charging station models generally use the centroids of polygons to represent the demand location. This approach inevitably leads to PCP, which is shown in the sixth column. The last column shows that except Liu (2012)'s model, all the models are designed for single mode charging.

To design an EV charging network, the proposed method should firstly be able to include both the fast charging stations (for short time charging need) and slow charging stations (for longer time charging need). Secondly, given the high cost of installing public charging stations, the model should be particularly budget-sensitive. In addition to $\$ 60,000-\$ 100,000$ for a Level 3 charging post and $\$ 3,500-\$ 6,000$ for a commercial Level 2 charging post, one would also have to consider the cost of renting/purchasing land for the station and other construction and operation costs (Community Energy Association, 2013). Moreover, to solve the range anxiety problem with a limited budget, the charging facilities should be accessible to as many EVs as possible. Thirdly, to ensure accuracy, network/polygons should be used to estimate fast/slow charging demand and the PCP must be resolved. Last but not least, to estimate charging demand more accurately, trip generation method should be used to estimate the demand for Level 2 charging and traffic assignment should be used to estimate the demand for Level 3 charging. 
Table 2.1: A classification of existing works

\begin{tabular}{|c|c|c|c|c|c|c|}
\hline Author(s) & $\begin{array}{l}\text { Demand } \\
\text { Model }\end{array}$ & $\begin{array}{l}\text { Decision } \\
\text { Model }\end{array}$ & Objective & $\begin{array}{l}\text { Demand } \\
\text { Repre- } \\
\text { senta- } \\
\text { tion }\end{array}$ & $\begin{array}{l}\text { Partial } \\
\text { Cov- } \\
\text { erage } \\
\text { Exists }\end{array}$ & $\begin{array}{l}\text { Station } \\
\text { Type }\end{array}$ \\
\hline $\begin{array}{l}\text { Chen et al. } \\
(2013)\end{array}$ & Regression & Optimization & $\begin{array}{l}\text { Minimize total ac- } \\
\text { cess cost }\end{array}$ & Point & YES & $\begin{array}{l}\text { Slow } \\
\text { Charg- } \\
\text { ing }\end{array}$ \\
\hline $\begin{array}{l}\text { Chen et al. } \\
(2014)\end{array}$ & $\begin{array}{l}\text { Traffic As- } \\
\text { signment }\end{array}$ & Ad hoc & $\begin{array}{l}\text { Minimize } \\
\text { travel time }\end{array}$ & Network & $\mathrm{N} / \mathrm{A}$ & $\begin{array}{l}\text { Fast } \\
\text { Charg- } \\
\text { ing }\end{array}$ \\
\hline $\begin{array}{l}\quad \text { Hanabusa } \\
\text { and } \\
\text { Horiguchi } \\
(2011)\end{array}$ & $\begin{array}{l}\text { Traffic As- } \\
\text { signment }\end{array}$ & Optimization & $\begin{array}{l}\text { Minimize } \\
\text { travel total } \\
\text { equalize electric } \\
\text { loads }\end{array}$ & Network & $\mathrm{N} / \mathrm{A}$ & $\begin{array}{l}\text { Fast } \\
\text { Charg- } \\
\text { ing }\end{array}$ \\
\hline $\begin{array}{l}\text { Frade et al. } \\
(2011)\end{array}$ & Regression & Optimization & $\begin{array}{l}\text { Maximize covered } \\
\text { demand }\end{array}$ & Point & YES & $\begin{array}{l}\text { Slow } \\
\text { Charg- } \\
\text { ing }\end{array}$ \\
\hline$\underset{(2011)}{\mathrm{Ge}}$ et al. & Ad hoc & Optimization & $\begin{array}{l}\text { Minimize charging } \\
\text { cost }\end{array}$ & Network & $\mathrm{N} / \mathrm{A}$ & $\begin{array}{l}\text { Fast } \\
\text { Charg- } \\
\text { ing }\end{array}$ \\
\hline$\underset{(2013)}{\mathrm{Xi}}$ et al. & Regression & Optimization & $\begin{array}{l}\text { Maximize charging } \\
\text { post usage }\end{array}$ & Point & YES & $\begin{array}{l}\text { Slow } \\
\text { Charg- } \\
\text { ing }\end{array}$ \\
\hline$\underset{(2014)}{\operatorname{Lam}}$ et al. & Ad hoc & Optimization & Minimize cost & Network & $\mathrm{N} / \mathrm{A}$ & $\begin{array}{l}\text { Fast } \\
\text { Charg- } \\
\text { ing }\end{array}$ \\
\hline $\begin{array}{l}\text { Lee et al. } \\
(2014)\end{array}$ & $\begin{array}{l}\text { Traffic As- } \\
\text { signment }\end{array}$ & Optimization & $\begin{array}{l}\text { Minimize network } \\
\text { cost and trip failure }\end{array}$ & Network & $\mathrm{N} / \mathrm{A}$ & $\begin{array}{l}\text { Fast } \\
\text { Charg- } \\
\text { ing }\end{array}$ \\
\hline Liu (2012) & Ad hoc & Ad hoc & $\begin{array}{l}\text { Minimize number } \\
\text { of charging stations }\end{array}$ & Polygon & $\mathrm{N} / \mathrm{A}$ & $\begin{array}{l}\text { Fast and } \\
\text { Slow } \\
\text { Charg- } \\
\text { ing }\end{array}$ \\
\hline
\end{tabular}




\section{Chapter 3}

\section{Partial Coverage Problem}

It is common practice to represent demand as discrete points in many location models for the convenience of calculating the distance between demands and servers (Miller, 1996). This approach of demand abstraction is easy to implement but too simplistic because it ignores the geometric characteristics of the demand, which could lead to problems and significant error in the result. The point abstraction only allows the demand to be either fully covered or not covered by a facility and does not account for partial coverage. This may result in partial coverage for geometric objects, which is called the partial coverage problem (PCP). PCP is illustrated in Figure 3.1 and Figure 3.2.

A point on a network is considered covered by a charging station only if the shortest network distance between this point and the charging station is less than the EV driving range allowed. If all points of a link are covered by a charging station, then that link is considered to be fully covered. Otherwise, the link is only partially covered. In Figure 3.1, the link EF is covered by charging station C2, link GH covered by charging station $\mathrm{C} 1$, and the middle link FG is not covered by any of the 


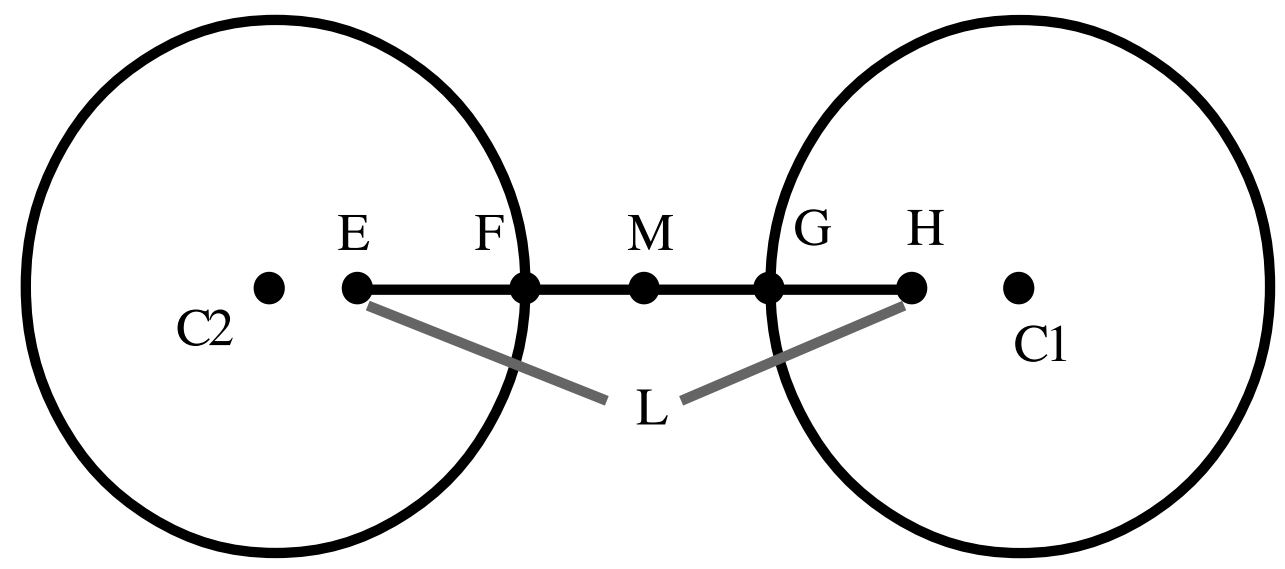

Figure 3.1: Partial coverage problem for lines

two charging stations. If we represent this road link by its middle point $\mathrm{M}$, then road link L is not covered by any charging stations, which is inaccurate.

Likewise, a point in a zone is considered covered by a charging station if and only if the Euclidean distance between this point and the charging station is less than the maximum walking distance. If all points in a zone are covered by a charging station, then the zone is considered to be fully covered. This situation is illustrated in Figure 3.2. The TAZ D is partially covered by charging stations $\mathrm{C} 1$ and $\mathrm{C} 2$. The left part of TAZ D is covered by charging station $\mathrm{C} 2$, the right part of $\mathrm{D}$ is covered by charging station $\mathrm{C} 1$, and the middle part of $\mathrm{D}$ is not covered by either charging station. If we use the centroid $\mathrm{M}$ to represent polygon $\mathrm{D}$, then $\mathrm{D}$ is not covered at all by any 


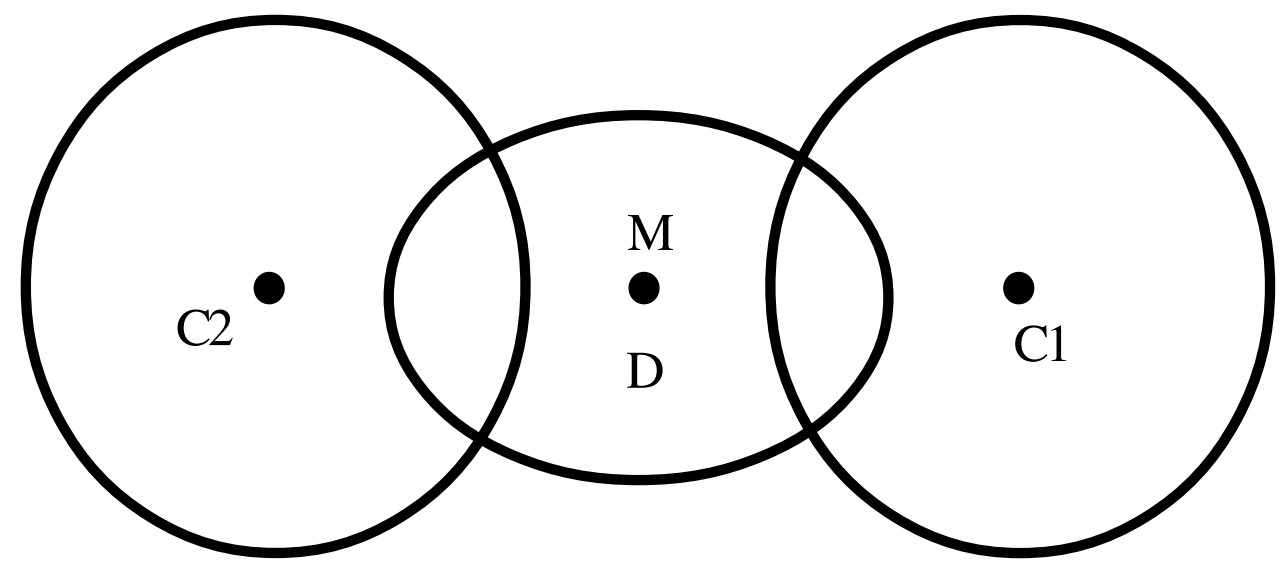

Figure 3.2: Partial coverage problem for polygons

charging station, which is inaccurate as well.

Aside from PCP, location models using point abstraction also suffer from MAUP, which means using different spatial units or scales for the same demand region can result in different solutions (Murray, 2005; Wong, 2009)

To address the problems caused by simplified point representation of demand in existing Set-Covering Problem (SCP), Murray (2005) develops a new SCP model using spatial objects (points, lines, polygons) to represent demand instead of point representation. In Murray (2005)'s model, complementary partial coverage (CP) by more than a given number of facilities is accounted as complete coverage. Alexandris and Giannikos (2010) extend Murray (2005)'s CP approach, applying it to the 
M.Sc. Thesis - Xiaozhou Zhang McMaster - Computational Science and Engineering

Maximum Covering Location Problem (MCLP). On the basis of Murray (2005)'s CP approach, Alexandris and Giannikos (2010) introduce penalty to complementary partial coverage, given that in many situations, Murray (2005)'s model may lead to redundant non-complementary coverage. An example of redundant partial coverage is given in Figure 3.3. Both circles cover more than half of square $\mathrm{S}$ but together don't cover the whole square area.

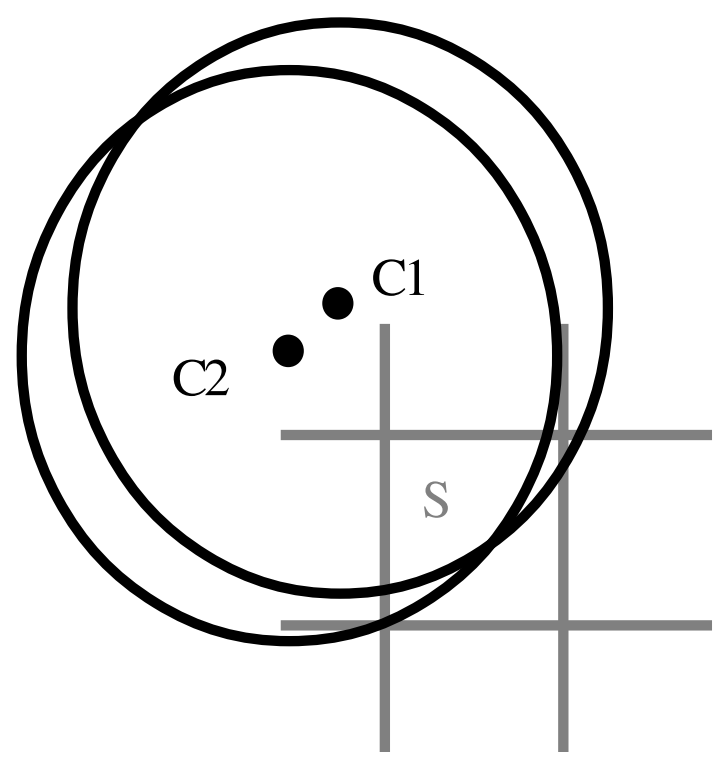

Figure 3.3: Redundant non-complementary partial coverage

Murray (2005)'s and Alexandris and Giannikos (2010)' models can improve the solution quality of SCP and MCLP. But their models are still subject to PCP and MAUP. Indeed, redundant non-complementary coverage still widely exists in their results, thus leading to more sites chosen than needed. In addition, their models 
M.Sc. Thesis - Xiaozhou Zhang McMaster - Computational Science and Engineering

cannot accurately evaluate the level of coverage.

To assess the performance of Murray (2005)'s CP approach on the charging station location problem, an optimization problem for EV charging stations incorporating the CP approach is formulated. This model resembles Murray (2005)'s model, minimizing total cost while guaranteeing the least level of coverage; it also introduces Alexandris and Giannikos (2010)' idea of penalty on partial coverage. The formulation of the modified model is as follows.

\section{Indices:}

$i$ index for polygons split from TAZs / index for links split from road networks $j$ index for candidate charging station locations

\section{Parameters:}

$d_{i j}$ the Euclidean distance from the candidate location $j$ to the furthest point in polygon $i$ or the network distance between the candidate location $j$ and the furthest point on link $i$

$w_{i}$ the number of EVs in polygon $i$ or the volume of road link $i *$ the length of road link $i$ multiplying the length of road link $i$

$R$ the maximum walking distance that $\mathrm{EV}$ drivers are willing to walk from charging station to destination or the driving range of EVs with $10 \%$ battery level (we assume that EV drivers will be alerted for charging when the battery level drops to $10 \%$ of the total capacity)

$c_{j}$ the cost for locating a charging station at location $j$

$B$ the budget for installing charging stations

$\alpha$ the least percentage of coverage 
M.Sc. Thesis - Xiaozhou Zhang McMaster - Computational Science and Engineering

$\beta$ the penalty coefficient for multiplying partially covered demanddemand partially covered by at least $\theta$ number of charging stations and not fully covered by any charging station

$\omega$ the least level of coverage that will be considered in complementary partial coverage

$\theta$ the least number of partial coverage needed to be treated as full coverage

\section{Sets:}

I set of polygons or road links

$J$ set of all candidate charging locations

$N(i)$ set of charging locations that can cover polygon $i$ or link $i, N(i)=\left\{j \mid d_{i j} \leq\right.$ $R\}$

$W(i)$ set of charging locations that can partially cover polygon or link $i$ by $\omega$

$M(j)$ set of polygons or links that are covered by charging station at $j, M(j)=$ $\left\{i \mid d_{i j} \leq R\right\}$

\section{Decision Variables:}

$x_{j}$ binary; $x_{j}=1$ if and only if a charging station is located in location $j$

$y_{i}$ binary; $y_{i}=1$ if and only if $i$ is covered by at least one charging station

$v_{i}$ binary; $v_{i}=1$ if and only if $i$ is partially covered by at least $\theta$ charging stations

\section{Model CP:}


$\underline{\text { M.Sc. Thesis - Xiaozhou Zhang McMaster - Computational Science and Engineering }}$

$$
\begin{array}{lll}
\min & \sum_{j \in J} c_{j} x_{j} & \\
\text { s.t. } & y_{i} \leq \sum_{j \in N(i)} x_{j} & \forall i \in I \\
& \theta \cdot v_{i} \leq \sum_{j \in W(i)} x_{j} & \forall i \in I \\
& y_{i}+v_{i} \leq 1 & \\
& \sum_{i \in I} w_{i}\left(y_{i}+\beta v_{i}\right) \geq \alpha \sum_{i \in I} w_{i} & \\
& x_{j} \in\{0,1\} & \forall j \in J \\
& y_{i}, v_{i} \in\{0,1\} & \forall i \in I
\end{array}
$$

The objective (1) is to minimize the total land cost for locating the charging stations. Constraint (2) guarantees that $i$ is considered fully covered if there is at least one charging station located and can cover $i$. Constraint (3) ensures that $i$ is considered complementarily partially covered if there are at least $\theta$ charging stations that each station can partially cover $i$ at least at $\omega$ level of coverage. Constraint (4) states that $i$ can only be either fully covered or complimentarily partially covered in order to avoid redundant coverage. Constraint (5) makes sure that at least $\alpha$ level of total demand should be covered in this model. Parameter $\beta$ (value no greater than 1) means a demand $i$ that is partially covered by multiple charging stations is regarded as equal to or less than complete coverage. When $\beta<1$, it is a penalty to multiple partial coverage to reduce non-complementary partial coverage since the model cannot accurately identify if multiple partial coverage is complementary or redundant. Constraint (6) and (7) ensure the decision variables are binary. In Section 6, we will apply this model to GTHA and Downtown Toronto for fast and slow 
M.Sc. Thesis - Xiaozhou Zhang McMaster - Computational Science and Engineering

charging stations respectively, denoted by FC-CP for fast charging station and SC$\mathrm{CP}$ for slow charging stations.

Since Murray (2005)'s and Alexandris and Giannikos (2010)' approach cannot completely solve PCP, we will introduce a geometric segmentation (GS) approach to eliminate PCP in Section 4 and 5. 


\section{Chapter 4}

\section{Fast Charging Stations}

In this chapter, we develop a model with geometric segmentation (FC-GS) to determine the location of the fast charging stations. In previous works that are based on network data, Flow Capturing Location Model (FCLM) and its extensions (Hodgson, 1990; Lee et al., 2014) have been applied to address fast charging station location problem. However, in FCLM, a traffic flow is considered as captured if there is at least one facility located on the path from its origin to the destination; while in FC-GS, a traffic link is considered as covered if it is within a certain range of at least one facility. FC-GS is more appropriate compared to FCLM for locating EV fast charging stations for the following reasons.

Firstly, FC-GS makes sure that EVs will have access to a nearest charging station within a given driving range while FCLM does not. According to previous research (National Renewable Energy Laboratory, 2014), slow charging modes (Level 1 \& 2) take up more than $90 \%$ of the EV charging demand and is sufficient for daily trips in most scenarios. As a solution to range anxiety, fast charging stations provide a reasonable option for urgent, unplanned charging needs. In this situation, a fast 
M.Sc. Thesis - Xiaozhou Zhang McMaster - Computational Science and Engineering

charging station serves as an emergency facility, which requires high accessibility. FC-GS will guarantee that EVs can have access to a nearest fast charging station within a given driving range, whereas FCLM has no such guarantee. Planners can even adjust the level of coverage according to their needs. Moreover, FCLM can lead to the undesirable scenario such that fast stations concentrate in the denser part of the road network, and EVs on other parts of the road network may not be able to reach the charging stations when needed.

Secondly, FC-GS is more practical to implement than FCLM. In an FCLM, facilities can only be placed at the nodes of the road network. But in reality, a facility is often placed at a distance from the road (off the network link). If the facility is close enough to a road, it can be approximated as a node in FCLM. However, when a facility is placed far away from a road, it is difficult to decide if it can capture the flow on the road. FC-GS does not have this problem since there is no limit on the location of the charging stations.

\subsection{Framework}

The framework of our model consists of five modules: data collection, geometric segmentation, traffic assignment, facility location optimization and charging load estimation.

Data used as model input include the road network, candidate sites for charging stations, the cost of each site, and data needed for traffic assignment.

Geometric segmentation is particularly noteworthy. In Figure 4.4, the left part of link $\mathrm{L}$ is covered by charging station $\mathrm{C} 2$, the right part of $\mathrm{L}$ is covered by charging station $\mathrm{C} 1$, and the middle part of $\mathrm{L}$ is not covered by any of the two charging 
M.Sc. Thesis - Xiaozhou Zhang McMaster - Computational Science and Engineering

stations. To avoid partial coverage, we can split link L into link L1, L2 and L3. After the split, L1 and L3 are fully covered by C2 and C1 while L2 is not covered. Therefore, the original road network can be transformed to a new network after the coverage segmentation operation. In the new network, partial coverage does not exist: A link is either completely covered or completely uncovered.

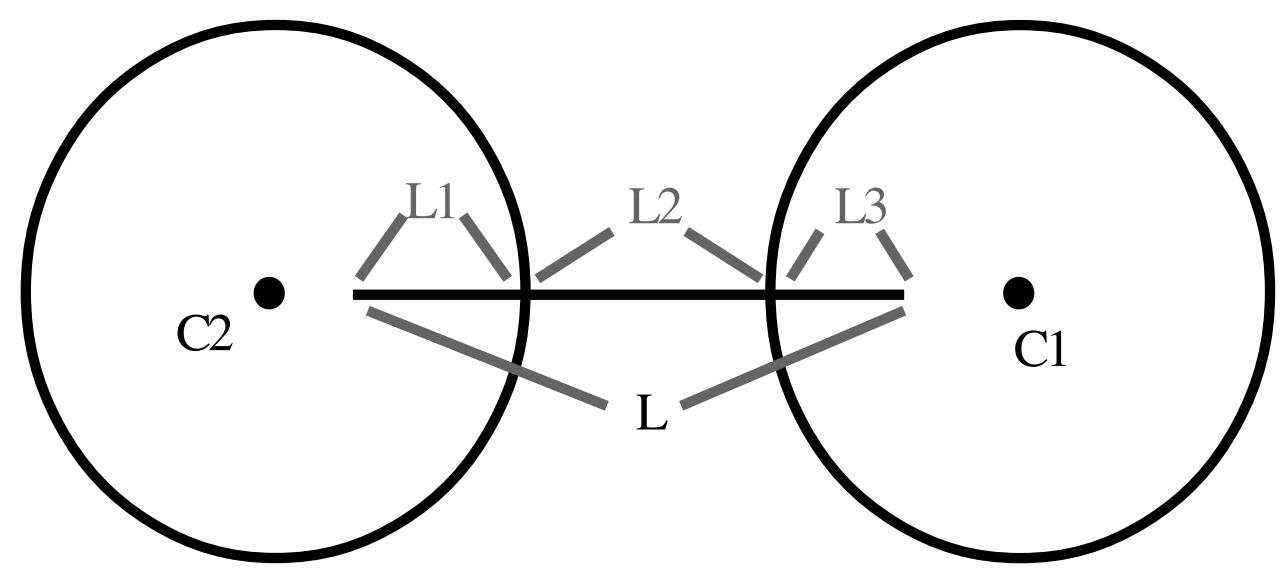

Figure 4.4: Splitting partially covered link

Traffic assignment is a common method used to forecast traffic in transportation. It allocates travel demand (origin-destination matrices) to a road network according to assignment rules. At the end of the assignment procedure, the whole traffic network reaches equilibrium. Then the traffic volume can be calculated for each link (Lam and Lo, 2004; Sheffi, 1995). 
M.Sc. Thesis - Xiaozhou Zhang McMaster - Computational Science and Engineering

Charging load estimation refers to how much charging demand goes to a certain charging station. This involves splitting charging demand covered by more than one charging station and summing up covered demand for each charging station. The splitting of charging demand can be achieved by using Network Analysis and Intersect function in ArcGIS, a Geographic Information System (GIS) software for mapping and spatial analysis, developed by Environmental Systems Research Institute (ESRI).

The details of the facility location optimization model for fast charging stations are presented in the next subsection.

\subsection{Optimization Model}

Model FC-GS is a variation of the classical maximum coverage problem with an objective to minimize the total installation cost while maintaining given percentage of coverage.

\section{Indices:}

$i$ index for road links

$j$ index for candidate charging station locations

\section{Parameters:}

$l_{i}$ the length of link $i$

$d_{i j}$ the shortest network distance from $i$ to $j$

$w_{i}$ the traffic volume of link $i$

$R$ the planned coverage range of the charging stations

$c_{j}$ the cost for locating a charging station at $j$

$B$ the budget limit for installing charging stations 
$\underline{\text { M.Sc. Thesis - Xiaozhou Zhang McMaster - Computational Science and Engineering }}$

$\alpha$ the least percentage of coverage

\section{Sets:}

$I$ set of links

$J$ set of all candidate charging locations

$N(i)$ set of charging locations that can cover link $i, N(i)=\left\{j \mid d_{i j} \leq R\right\}$

$M(j)$ set of links that are covered by the charging station located at $j, M(j)=$ $\left\{i \mid d_{i j} \leq R\right\}$

\section{Decision Variables:}

$x_{j}$ binary; $x_{j}=1$ if and only if a charging station is located at $j$

$y_{i}$ binary; $y_{i}=1$ if and only if road link $i$ is covered by at least one charging station

\section{Model FC-GS:}

$$
\begin{array}{lll}
\min & \sum_{j \in J} c_{j} x_{j} & \\
\text { s.t. } & y_{i} \leq \sum_{j \in N(i)} x_{j} & \forall i \in I \\
& \sum_{i \in I} w_{i} l_{i} y_{i} \geq \alpha \sum_{i \in I} w_{i} l_{i} & \\
& x_{j} \in\{0,1\} & \forall j \in J \\
& y_{i} \in\{0,1\} & \forall i \in I
\end{array}
$$

Objective (1) is to minimize the total cost of charging stations. Constraint (2) states that $\mathrm{i}$ is considered covered if its demand is satisfied by at least one charging station. Constraint (3) guarantees that at least $\alpha$ portion of the coverage of the road network with traffic flow as the weight of each link. Normally we should use 
$\underline{\text { M.Sc. Thesis - Xiaozhou Zhang McMaster - Computational Science and Engineering }}$

the number of EVs on each link to represent the fast charging demand. However, since we can only calculate the traffic volume on each link, which means the same vehicle will be counted on each link that is on its path, we use the length of each link multiplied by the link traffic volume to represent the fast charging demand. It can effectively represent the weight of each link compared to other links. Constraint sets (4) and (5) guarantee that decision variables $x_{j}$ and $y_{i}$ are binary variables. 


\section{Chapter 5}

\section{Slow Charging Stations}

The maximum covering model for slow charging stations (SC-GS) is formulated in a similar way to the Model FC-GS for fast charging stations. The main difference is that for the slow charging model the demand for charging is based on TAZs rather than the network links that are used in the fast charging model. In other words, we assume that the slow charging demand arises from areas, instead of links.

We argue that planning for slow charging stations should be done based on the central district of a city rather than on the whole metropolitan area as with fast charging stations. There are two reasons for this choice. Firstly, the use of slow charging station is for drivers to charge their EVs at their trip destinations (excluding home), thus the slow charging stations should be located in places that people are more likely to visit, such as workplaces, shopping malls, theatres and restaurants. As a result, to ensure the cost-effectiveness of the level 2 charging stations, the less visited areas and areas without parking lots should be of low priority in slow charging station network design. Secondly, the range requirement of slow charging is different from that of fast charging. The EV drivers will be satisfied if they could find slow charging 
M.Sc. Thesis - Xiaozhou Zhang McMaster - Computational Science and Engineering

stations within a walking distance of their activity places. In our experiment, we will use 500 meters and 300 meters as the maximum covering ranges for a slow charging station, which we assume are the possible maximum walking distances a driver might be willing to walk from a charging station to the destination, or vice versa.

\subsection{Framework}

The framework of the model for slow charging stations contains the five modules: data collection, trip generation, geometric segmentation, facility location optimization and charging load estimation.

Data input for the model includes TAZ file, candidate sites for charging stations, cost of each site and data needed for demand estimation.

The determination of demand consists of estimating the number of EVs that go to a certain TAZ on a daily basis. The demand can be estimated through the trip generation method in the travel forecasting process. Trip generation uses the land use and demographic information to predict the total number of trips entering or leaving a zone in the city (Meyer and Miller, 1984).

Conceptually, the geometric segmentation of TAZs is similar to that of the road links in Section 3. The difference is that the geometric object we consider in this section is a polygon and not a line. Specifically, the covering range of a charging station is a circular area with the allowed maximum walking distance as its radius. This case is illustrated in Figure 5.5. The TAZ D is partially covered by charging stations $\mathrm{C} 1$ and $\mathrm{C} 2$. The left part of TAZ D is covered by charging station $\mathrm{C} 2$, the right part of $\mathrm{D}$ is covered by charging station $\mathrm{C} 1$, and the middle part of $\mathrm{D}$ is not covered by either charging station. To avoid partial coverage, we can split D into D1, 
M.Sc. Thesis - Xiaozhou Zhang McMaster - Computational Science and Engineering

D2 and D3. After the split, D2 and D3 are fully covered by C2 and C1 while D1 is not covered.

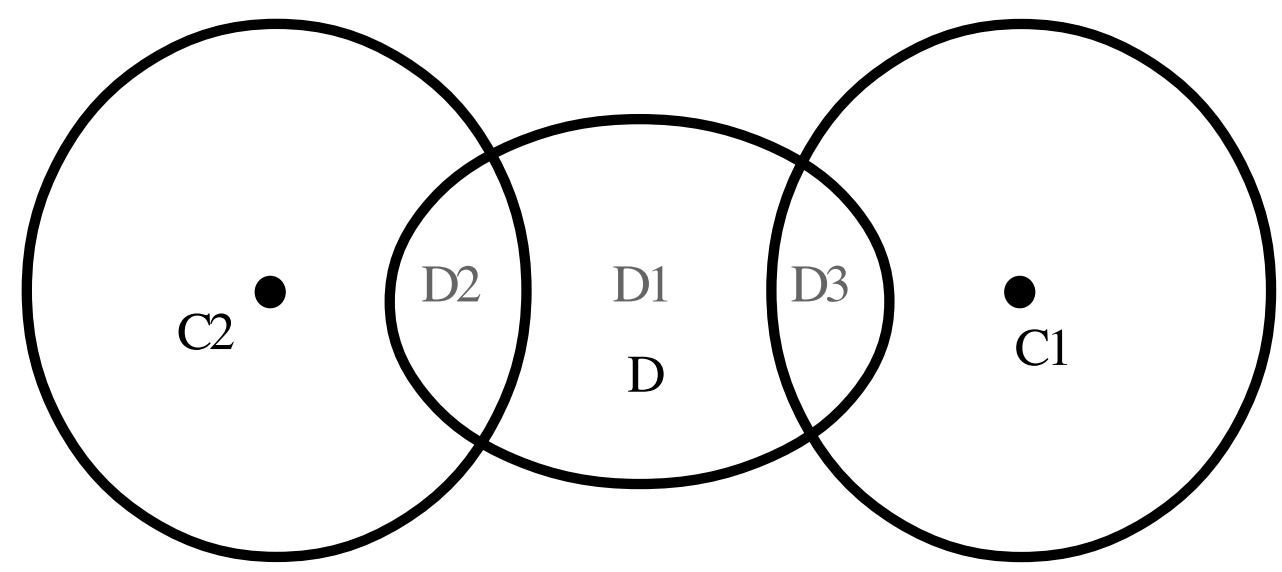

Figure 5.5: Splitting partially covered TAZ

Charging load estimation for slow charging stations is similar to that for fast charging stations. The splitting of commonly covered TAZ can be achieved by dissecting the commonly covered area with Thiessen Polygon generated from the chosen charging station locations in ArcGIS.

We will present the facility location optimization model in the next subsection. 
M.Sc. Thesis - Xiaozhou Zhang McMaster - Computational Science and Engineering

\subsection{Optimization Model}

\section{Indices:}

$i$ index for polygons split from TAZs

$j$ index for candidate slow charging station locations

\section{Parameters:}

$d_{i j}$ the Euclidean distance from the candidate location $j$ to the furthest point in polygon $i$

$w_{i}$ the number of EVs in polygon $i$

$R$ the maximum walking distance which one is willing to walk from charging station to destination, or vice versa

$c_{j}$ the cost for locating a charging station at $j$

$B$ the budget limit for installing charging stations

$\alpha$ the least percentage of coverage

\section{Sets:}

$I$ set of polygons

$J$ set of all candidate charging locations

$N(i)$ set of charging locations that can cover polygon $i, N(i)=\left\{j \mid d_{i j} \leq R\right\}$

$M(j)$ set of polygons that are covered by the charging station located at $j, M(j)=$ $\left\{i \mid d_{i j} \leq R\right\}$

\section{Decision Variables:}

$x_{j}$ binary; $x_{j}=1$ if and only if a charging station is located at $j$

$y_{i}$ binary; $y_{i}=1$ if and only if polygon $i$ is covered by at least one charging station 
M.Sc. Thesis - Xiaozhou Zhang McMaster - Computational Science and Engineering

\section{Model SC-GS:}

$$
\begin{array}{lll}
\min & \sum_{j \in J} c_{j} x_{j} & \\
\text { s.t. } & y_{i} \leq \sum_{j \in N(i)} x_{j} & \forall i \in I \\
& \sum_{i \in I} w_{i} y_{i} \geq \alpha \sum_{i \in I} w_{i} & \\
& x_{j} \in\{0,1\} & \forall j \in J \\
& y_{i} \in\{0,1\} & \forall i \in I
\end{array}
$$

Clearly, Model SC-GS for slow charging stations is developed by geometric object segmentation as in the Model FC-GS for fast charging stations. The differences are: 1) The former is by segmentation of polygons, while the latter is by segmentation of road links. In other words, $I$ represents links in Model FC-GS but polygons in Model SC-GS; 2) $d_{i j}$ represents the Euclidean distance between demand and charging station in Model SC-GS while it represents length of the shortest path between demand and charging station in Model FC-GS; 3) $R$ is the maximum walking distance between demand and charging station in Model SC-GS while it represents the minimum driving range of EVs to be able to reach a closest charging station; 4) Model FC-GS uses the product of link length and link flow quantity as an estimation of the demand of each link, while Model SC-GS uses the number of EVs in each zone as the demand. 


\section{Chapter 6}

\section{Numerical Experiments}

\subsection{Fast Charging Stations}

Both model FC-CP and model FC-GS are applied to the Greater Toronto and Hamilton Area (GTHA) road network. The candidate locations are chosen from 657 gas stations in the GTHA area. We use the average housing price of the census tracts to which each candidate site belongs to represent the cost of locating a charging station at these sites. The original GTHA road network consists of 2511 links. We use traffic flow data generated directly from TRAFFIC, a model that was developed at the McMaster Institute for Transportation and Logistics (MITL) and has been used extensively to estimate emissions from traffic flows for several Canadian cities. (Rashidi et al., 2015; Maoh and Kanaroglou, 2009; Buliung et al., 2005).

For the model FC-CP, we set $\beta=1, \omega=50 \%, \theta=2$, which means one road link with partial coverage over $50 \%$ from 2 stations can be regarded as full coverage without any penalty. Our experiment shows that any values of $\beta<1$ and $\omega<50 \%$ lead to larger errors in the result or even no feasible solution. Model FC-CP for fast 
$\underline{\text { M.Sc. Thesis - Xiaozhou Zhang McMaster - Computational Science and Engineering }}$

Table 6.2: Model sizes using different ranges (fast charging)

\begin{tabular}{lrrr}
\hline Model & Cov. Range $(\mathrm{km})$ & Dec. Var. \# & Cons. \# \\
\hline & Before Splitting & 3168 & 2512 \\
& 5 & 20032 & 19376 \\
FC-GS & 10 & 37902 & 37246 \\
& 15 & 52758 & 52102 \\
& 20 & 64249 & 63593 \\
\hline FC-CP & $5 / 10 / 15 / 20$ & 5679 & 7534 \\
\hline
\end{tabular}

charging stations consists of 5679 decision variables and 7534 constraints.

For model FC-GS, we want to compare the results using range $R=5 \mathrm{~km}, 10 \mathrm{~km}$, $15 \mathrm{~km}, 20 \mathrm{~km}$ and coverage level $\alpha=85 \%, 90 \%, 95 \%, 99.9 \%$. Using different ranges to preprocess the road network data in ArcGIS, we get 19375 links from the original 2511 links using $R=5 \mathrm{~km}, 37245$ links for $R=10 \mathrm{~km}, 52101$ links for $R=15 \mathrm{~km}$ and 63592 links for $R=20 \mathrm{~km}$. The number of links increases significantly after splitting. Then the length of each new link is recalculated in ArcGIS. Table 6.2 shows the different model sizes using different ranges. The number of decision variables and constraints grows significantly with the increasing range. It is obvious that the size of model FC-GS increases with the range while the size of model FC-CP remains irrelevant to the range.

Using the Network Analysis module of ArcGIS, we get the coverage information from each location to each link. The models are implemented using CPLEX 12.6 API for Python and run on a Dell Latitude E5530 computer with Intel Core i7-3540M 3.00GHz CPU and 8GB memory.

Table 6.3 shows the computational results of the model FC-CP. Table 6.4 shows the computational results of the model FC-GS. The solution time is the time used by 
$\underline{\text { M.Sc. Thesis - Xiaozhou Zhang McMaster - Computational Science and Engineering }}$

Table 6.3: Computational results of Model FC-CP $(R=15 \mathrm{~km})$

\begin{tabular}{crrr}
\hline Targeted Cov. & Sol. Time (Min) & Station \# & True Cov. \\
\hline $85 \%$ & 5.83 & 17 & $90.03 \%$ \\
$90 \%$ & 22.28 & 22 & $92.55 \%$ \\
$95 \%$ & 8.24 & 28 & $96.87 \%$ \\
$99.9 \%$ & Infeasible & Infeasible & Infeasible \\
\hline
\end{tabular}

the solver to solve the Mixed Integer Linear Programming (MILP) problem. Table 6.3 shows that there is a great discrepancy between the targeted covering level in model FC-CP and the true covering level achieved by the optimal results of model FC-CP. The true coverage is either higher or lower than the targeted coverage. This is easily explained by Figure 6.6, where the two circles form complementary partial coverage on the elliptical area. When the combined area of D1 and D2 is greater/smaller than the combined area of D3 and D4, the resulting coverage will be greater/smaller than the targeted coverage. Table 6.4 shows that the number of charging stations needed decreases as the range increases, but increases with the level of coverage significantly the number of charging stations needed for $99.9 \%$ coverage is almost twice the number of stations needed for $95 \%$ coverage. So the marginal coverage increase by a charging station will decrease as the total number of charging stations increases. By comparing these two charts, we note that that the solution time is irrelevant to the model size. Obviously, for the same range and coverage level, model FC-CP requires much more number of charging stations than model FC.

We visualize the results of the two models with $R=15 \mathrm{~km}$ and $\alpha=90 \%$ in Figure 6.7, which compares the visualized results between model FC-GS and model FC-CP. The underlying green lines are the road network. The thickness of the line reflects 
M.Sc. Thesis - Xiaozhou Zhang McMaster - Computational Science and Engineering

Table 6.4: Computational results of Model FC-GS

\begin{tabular}{crrr}
\hline Cov. Range $(\mathrm{km})$ & Targeted Cov. & Sol. Time (Min) & Station \# \\
\hline \multirow{3}{*}{5} & $85 \%$ & 1.1 & 74 \\
& $90 \%$ & 0.4 & 111 \\
& $95 \%$ & Infeasible & Infeasible \\
& $99.9 \%$ & Infeasible & Infeasible \\
\hline \multirow{3}{*}{10} & $85 \%$ & 6.8 & 20 \\
& $90 \%$ & 2.9 & 26 \\
& $95 \%$ & 3.1 & 36 \\
15 & $99.9 \%$ & Infeasible & Infeasible \\
\hline & $85 \%$ & 7.1 & 10 \\
& $90 \%$ & 5.1 & 12 \\
20 & $95 \%$ & 1.4 & 17 \\
& $99.9 \%$ & 0.4 & 33 \\
\hline & $85 \%$ & 11.8 & 6 \\
& $90 \%$ & 6.3 & 7 \\
& $95 \%$ & 16.8 & 10 \\
& $99.9 \%$ & 1.6 & 19 \\
\hline
\end{tabular}




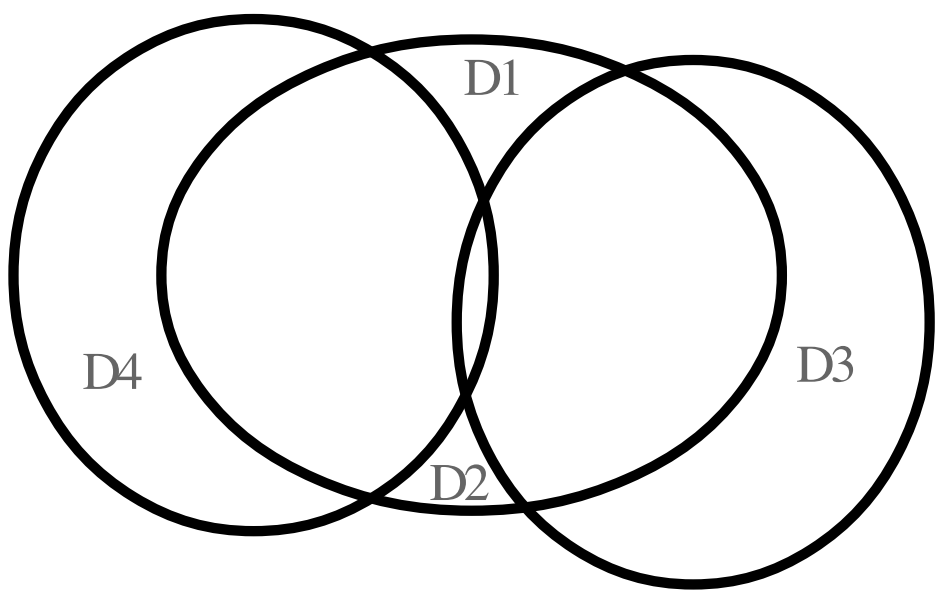

Figure 6.6: Coverage gap

the traffic volume on the road links. The blue lines are roads covered by charging stations. Both graphs show that the models tend to choose to cover roads with more traffic volume. By comparing the two graphs, it is noted that model FC-GS chooses much less charging stations than model FC-CP and the chosen stations in model FCGS are more evenly distributed than those chosen by model FC-CP, which contains several groups of charging stations that are very close to each other. This is a result of non-complimentary partial coverage.

The results show that the model FC-GS is more effective in determining the locations of fast charging stations. 

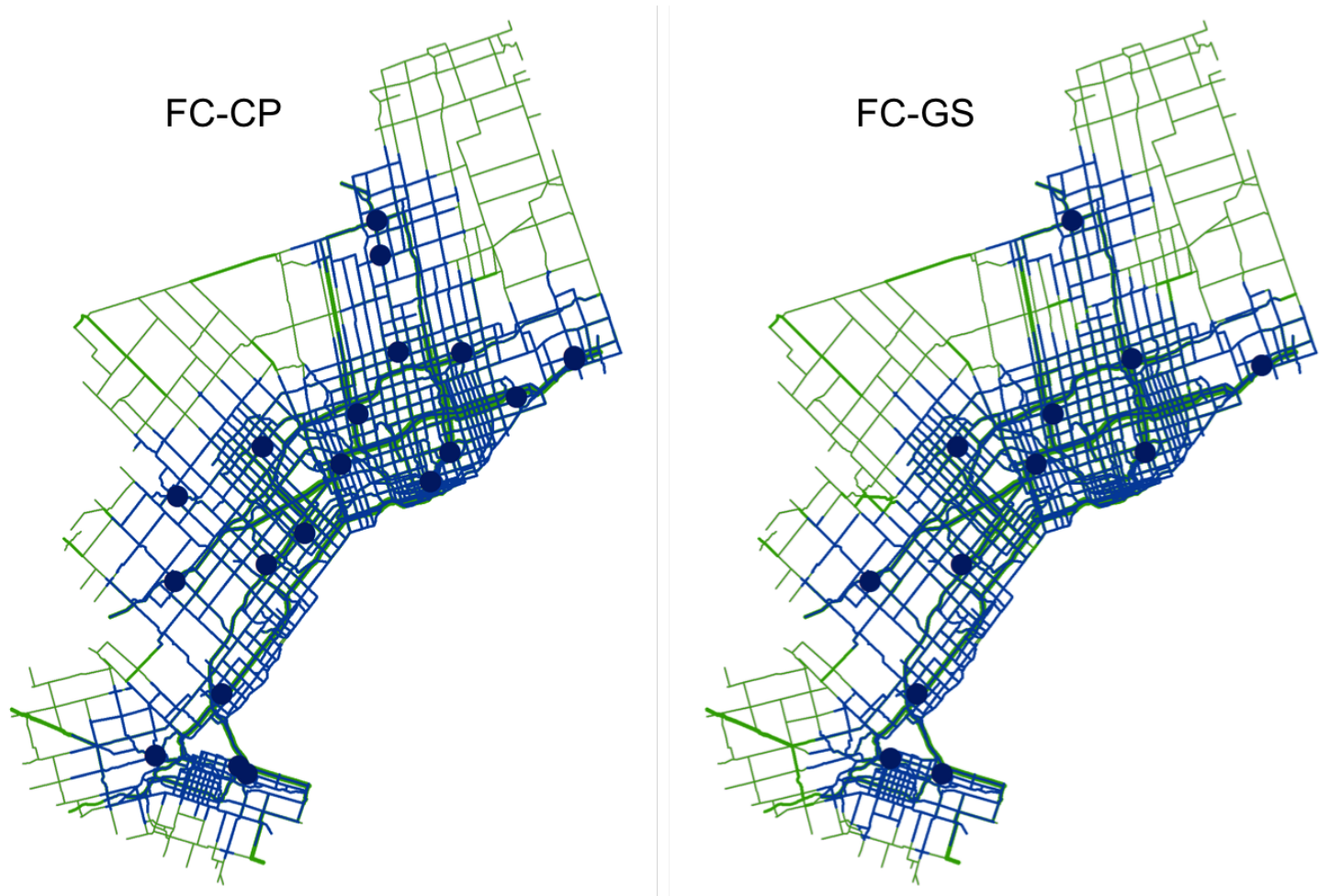

Figure 6.7: Comparison of visualized results $(\mathrm{R}=15 \mathrm{~km}$, alpha $=90 \%)$

\subsection{Slow Charging Stations}

Both the model SC-GS and the model SC-CP are applied to the Downtown Toronto area. The original Downtown Toronto area consists of 64 TAZs. Using the OriginDestination matrix we get from Traffic Model (McMaster Institute for Transportation and Logistics, 2014), we estimate how many vehicles will visit a TAZ in one day. By assuming a certain proportion of EVs among all vehicles, we can estimate the number of EVs that go to a TAZ on a daily basis. The 300 candidate locations are chosen from Downtown Torontos Points of Interest (POI), including restaurants, parking lots, schools and other businesses or public institutions in downtown. Similar to Model FC, we use the average housing price of the census tracts to which each candidate 
M.Sc. Thesis - Xiaozhou Zhang McMaster - Computational Science and Engineering

Table 6.5: Model sizes using different ranges (slow charging)

\begin{tabular}{crrr}
\hline Model & Cov. Range $(\mathrm{m})$ & Dec. Var. \# & Cons. \# \\
\hline & Before Splitting & 364 & 65 \\
SC-GS & 300 & 7566 & 7267 \\
& 500 & 17380 & 17081 \\
\hline SC-CP & $300 / 500$ & 428 & 193 \\
\hline
\end{tabular}

site belongs to represent the cost of locating a charging station at these sites.

Model SC-CP for slow charging stations consists of 428 decision variables and 193 constraints. We set $\beta=1, \omega=50 \%, \theta=2$ the same way as for fast charging stations. For $\beta$, a value less than 1 will lead to no solution for SC-CP and a value less than $50 \%$ for $\omega$ with a value more than 3 for $\theta$ will lead to more error and more charging stations selected. This situation is illustrated in the experiment results in Table A.10 and Figure A.13 in the Appendix.

For model SC-GS, we compare the results using different coverage level $\alpha=85 \%$, 90\%, 95\%, 99.9\%, as well. After segmentation, the TAZs are split into 7266 and 17080 polygons by the coverage areas of the candidate locations, which are circular areas with 300 and 500 meter radius respectively. Table 6.5 shows the different model sizes of different models with different ranges. The weight of each polygon is the number of EVs in each polygon. When splitting TAZ to polygons, EVs in each TAZ are assigned to each polygon, too. The number of EVs assigned to each polygon is proportional to its area.

The models are implemented in the same way and run on the same environment as Section 6.1.

Table 6.6 shows the computational results of the model SC-CP for slow charging 
$\underline{\text { M.Sc. Thesis - Xiaozhou Zhang McMaster - Computational Science and Engineering }}$

Table 6.6: Computational results of Model SC-CP $(R=500 \mathrm{~m})$

\begin{tabular}{cccc}
\hline Targeted Cov. & Sol. Time (Min) & Station \# & True Cov. \\
\hline $85 \%$ & 0.004 & 21 & $85.71 \%$ \\
$90 \%$ & 0.008 & 24 & $89.87 \%$ \\
$95 \%$ & 0.006 & 29 & $92.85 \%$ \\
$99 \%$ & 0.003 & 32 & $95.60 \%$ \\
\hline
\end{tabular}

Table 6.7: Computational Results of Model SC-GS

\begin{tabular}{crrr}
\hline Cov. Range $(\mathrm{m})$ & Targeted Cov. & Sol. Time (Min) & Station \# \\
\hline \multirow{3}{*}{300} & $85 \%$ & 27.9 & 37 \\
& $90 \%$ & 127.1 & 45 \\
& $95 \%$ & 69.9 & 54 \\
& $99 \%$ & 247.8 & 70 \\
\hline \multirow{3}{*}{500} & $85 \%$ & 6.4 & 13 \\
& $90 \%$ & 30.9 & 16 \\
& $95 \%$ & 379.1 & 19 \\
& $99 \%$ & 570.0 & 25 \\
\hline
\end{tabular}

stations. Due to its small problem size, the SC-CP for slow charging requires no time (less than 1 second) to be solved. But the great discrepancy between true coverage and targeted coverage level also exists in slow charging. Table 6.7 shows the computational results of model SC-GS. We note that both the number of charging stations and the solution time increase significantly with the increase of the percentage of coverage. Comparing Table 6.6 and Table 6.7, it is clear that model SC-CP requires more charging stations than model SC-GS.

Figure 6.8 shows the comparison of visualized results between model SC-CP and model SC-GS with $R=500 \mathrm{~m}$ and $\alpha=90 \%$. The areas in the circles are covered by selected charging stations. The models tend to choose to cover TAZs with more EVs 
M.Sc. Thesis - Xiaozhou Zhang McMaster - Computational Science and Engineering

(polygons of darker colors). Also, it is evident that the result of model SC-CP suffers from serious non-complementary partial coverage problem while the charging stations selected by model SC-GS are evenly distributed and much less than charging stations selected by SC-CP. It shows that model SC-GS is more effective in determining the location of slow charging stations.
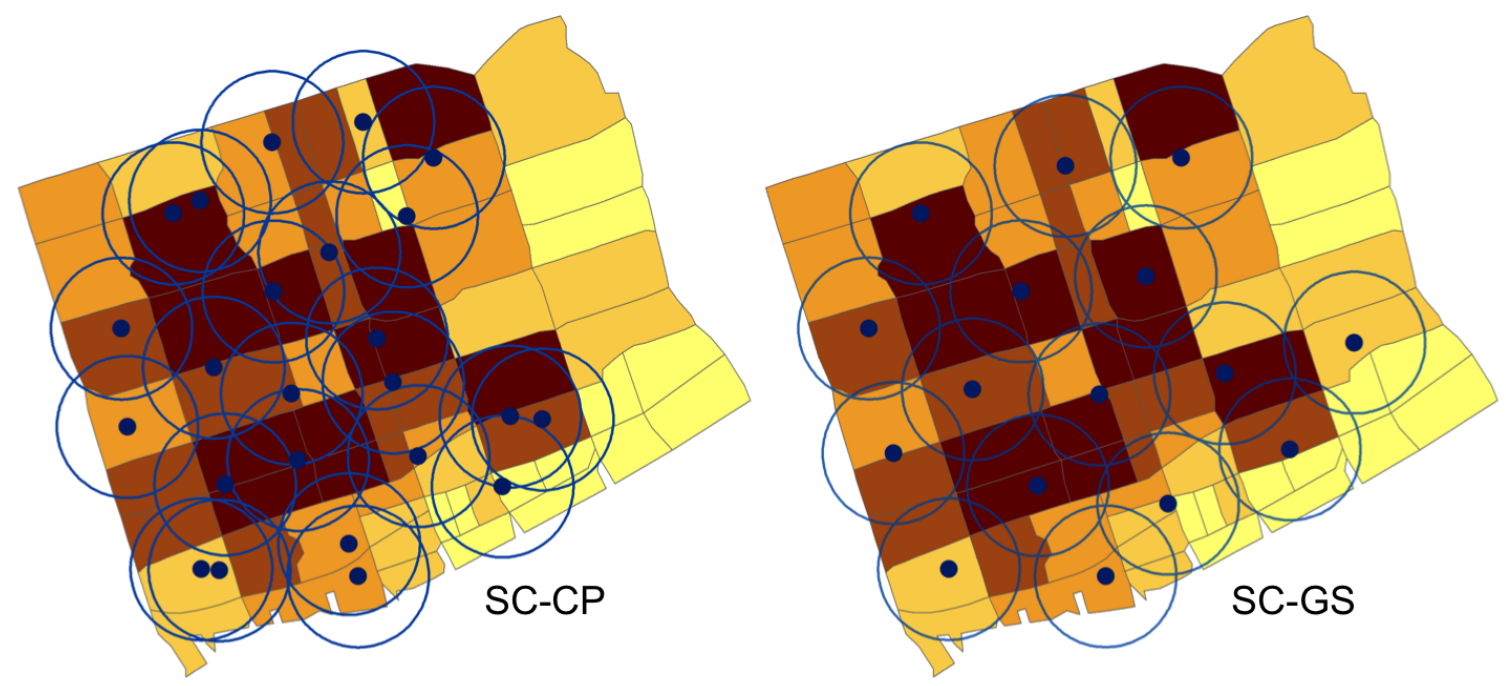

Figure 6.8: Comparison of visualized results $(\mathrm{R}=500 \mathrm{~m}$, alpha $=90 \%)$

\subsection{Estimating Demand for Each Station}

As we can see from the results of the experiment, facilities close to each other may have shared covered demand areas/links. How much of the demand should be assigned to each facility is a problem that needs to be addressed. We follow the rule that demand should be assigned to its nearest facility.

For fast charging stations, we use the Network Analysis module of ArcGIS to split the commonly covered demand network into different parts, and each part is 
M.Sc. Thesis - Xiaozhou Zhang McMaster - Computational Science and Engineering

assigned to one facility, ensuring that any point in the assigned link is closest to its corresponding facility compared to other facilities. In this way, we can get the nonoverlapping covered demand road links for each charging station. By assuming $10 \%$ EV market share, we get the number of EV kilometers each charging station covers. The result is illustrated in Figure 6.9.

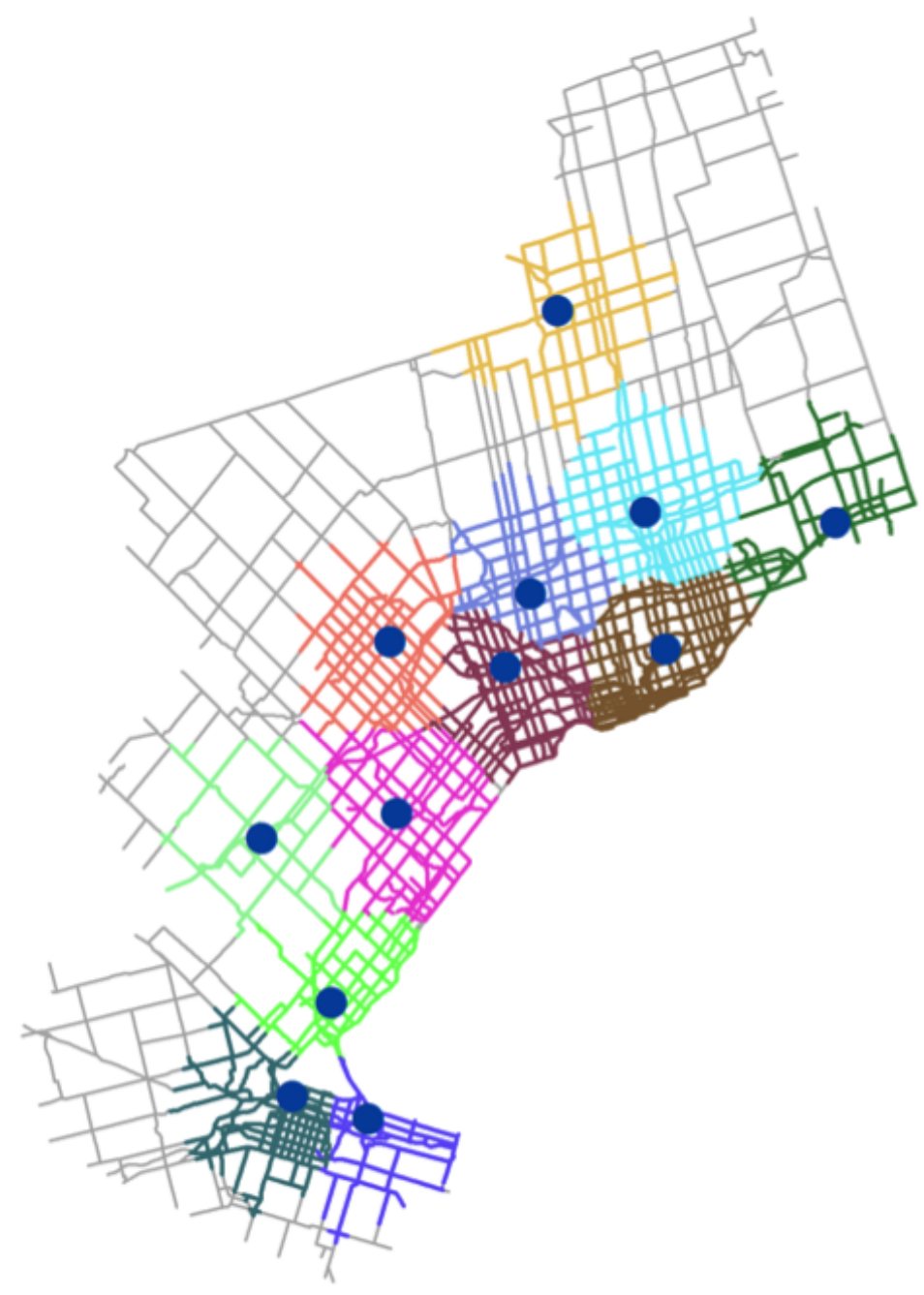

Figure 6.9: Divide fast charging demand

The estimation of charging demand for each fast charging station is listed in Table 
M.Sc. Thesis - Xiaozhou Zhang McMaster - Computational Science and Engineering

6.8.

For slow charging, we use Thiessen polygon generated from the selected charging station locations to split the shared covered demand. Thiessen polygons are polygons generated from a set of sample points on a plane. Each Thiessen polygon has a sample point inside itself. Any point inside a Thiessen polygon is closer to its sample point than any other sample point. The Thiessen polygons generated from the chosen charging station locations of model SC-GS are illustrated in Figure 6.10.

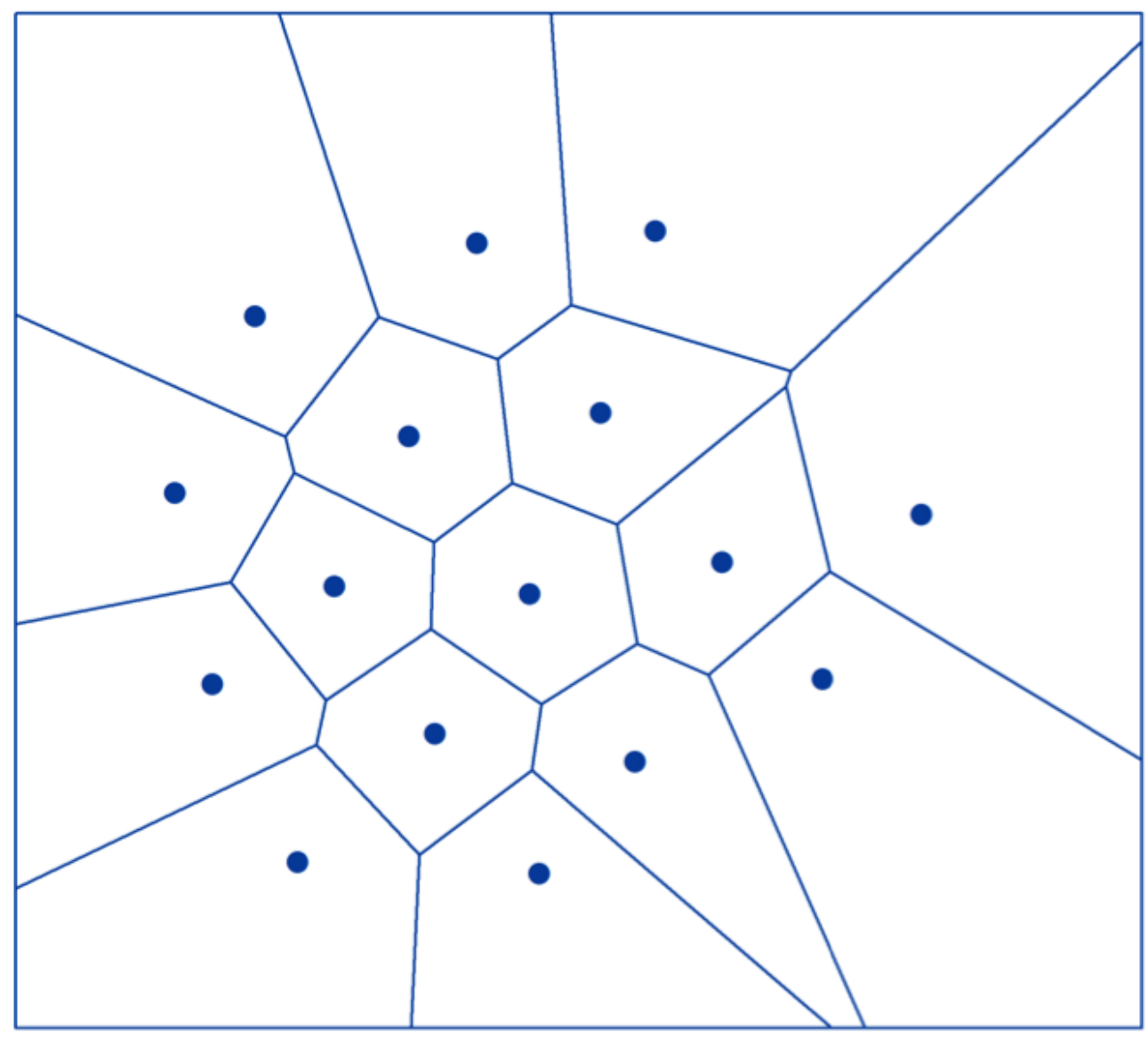

Figure 6.10: Thiessen polygons 
M.Sc. Thesis - Xiaozhou Zhang McMaster - Computational Science and Engineering

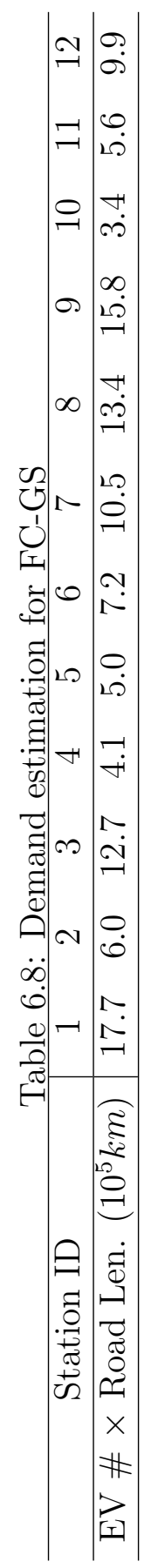


M.Sc. Thesis - Xiaozhou Zhang McMaster - Computational Science and Engineering

By intersecting Thiessen polygons with the covered demand region, we split the shared covered area into several equal parts and assign them to their closest charging stations. Figure 6.11 and 6.12 illustrate the process of using Thiessen polygons to divide the shared demand and assign the demand to each charging station. By assuming $10 \%$ EV market share, we get the number of EVs each charging station covers. This is shown in Table 6.9.

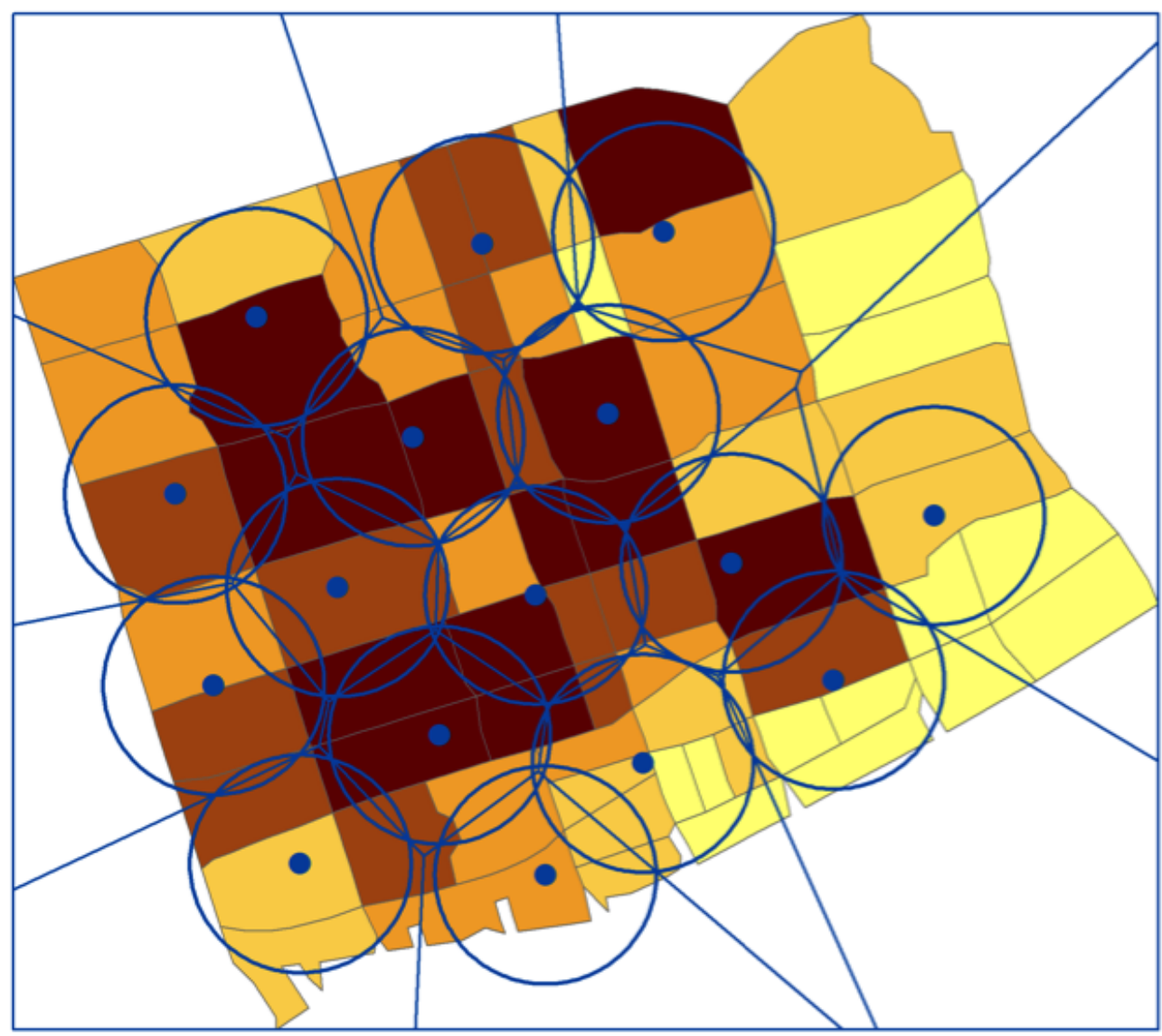

Figure 6.11: Thiessen polygons splitting shared demand 
M.Sc. Thesis - Xiaozhou Zhang McMaster - Computational Science and Engineering

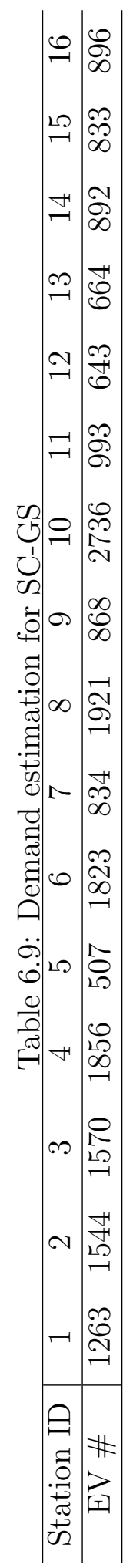




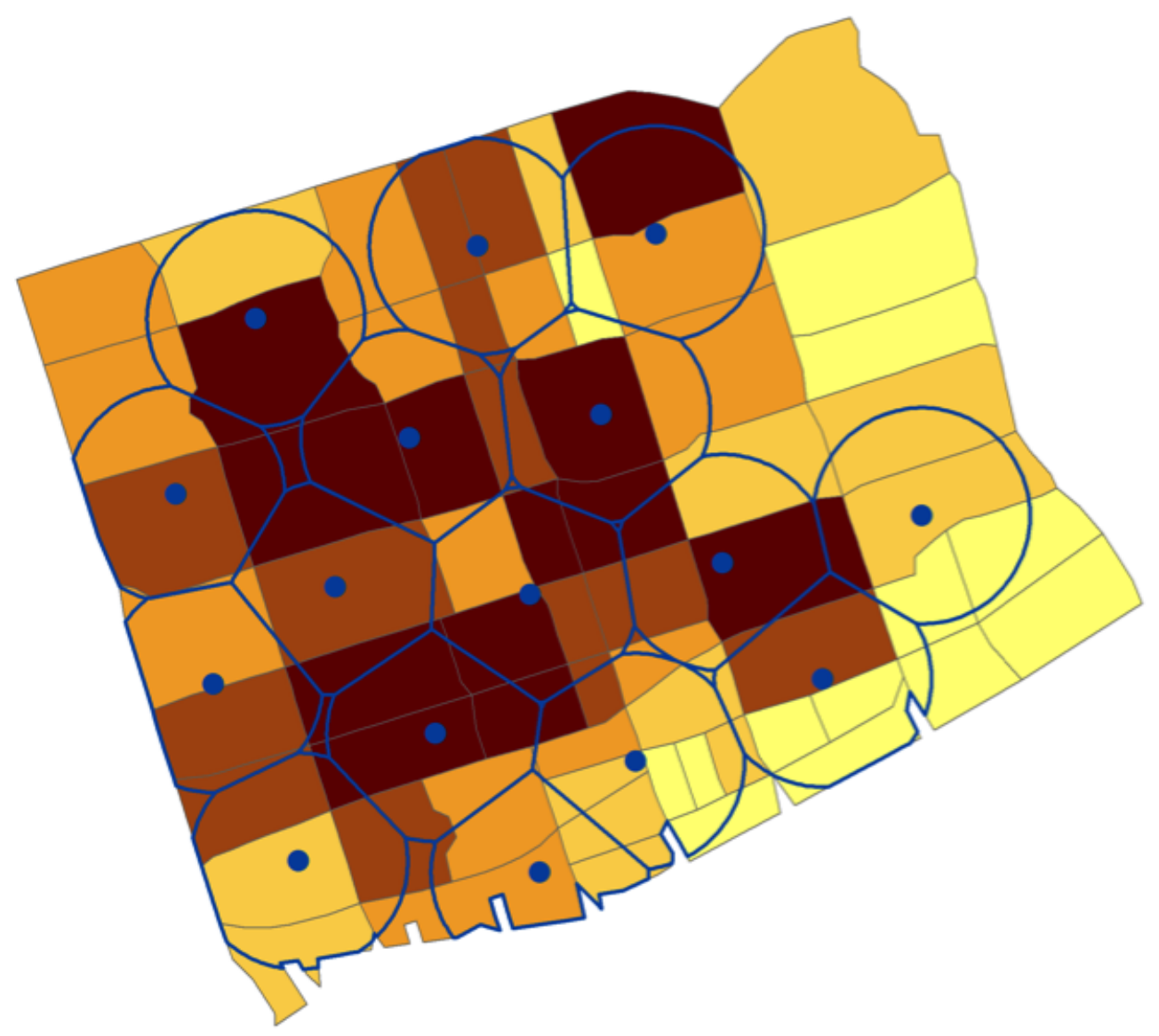

Figure 6.12: Divide slow charging demand 


\section{Chapter 7}

\section{Conclusion}

We proposed a method to locate both fast and slow charging stations to address different charging needs under the same framework. The models are designed to tackle range anxiety by minimizing the total cost while guaranteeing a level of demand coverage. The model can also be easily adapted by limiting the budget or the number of charging stations and maximizing the demand coverage. We use more realistic geometric objects - networks and polygons - to represent the charging demand instead

of using discrete points. Moreover, we solve the PCP by using geometric segmentation method.

The numerical study shows that the models are practical and effective in deciding the locations of fast and slow charging stations. The result shows that the charging stations have been evenly located in the urban areas. A comparison between the results of GS and CP shows that geometric segmentation can fully eliminate the partial coverage issue and produce much more accurate and reliable result than complementary partial coverage approach. Although the segmentation of the links/polygons significantly increases the problem size, the models remain solvable within reasonable 
$\underline{\text { M.Sc. Thesis - Xiaozhou Zhang McMaster - Computational Science and Engineering }}$

computation time.

In future research, this model can be improved in several directions. Firstly, the capacities of the charging stations can be introduced to ensure that charging demands can be fully served by their nearby charging stations, which arise from the capacities of the electricity grid network. Secondly, we already know that EV drivers travel behaviors affect the location decision of charging stations, but the locations of fast charging stations may affect the travel decisions of EV drivers as well. For example, long distance EV drivers may tend to choose traveling route by fast charging stations and EV drivers may also choose restaurants with slow charging stations installed when making their dining decisions. So we can combine the demand estimation and facility location optimization together. Thirdly, we may implement the model within ArcGIS to automate the whole process without intermediate steps. Last but not least, heuristics can be developed to solve this problem more efficiently. 


\section{Appendix A}

\section{Appendix}

Table A.10: Computational results for $\mathrm{SC}-\mathrm{CP}(\mathrm{R}=500 \mathrm{~m}$, alpha $=90 \%$, beta $=1$, omega $=33 \%$, theta $=3$ )

\begin{tabular}{cccc}
\hline Targeted Cov. & Sol. Time (Min) & Station \# & True Cov. \\
\hline $85 \%$ & 0.049 & 24 & $85.35 \%$ \\
$90 \%$ & 0.043 & 28 & $87.78 \%$ \\
$95 \%$ & 0.017 & 32 & $89.66 \%$ \\
$99 \%$ & 0.022 & 36 & $93.75 \%$ \\
\hline
\end{tabular}




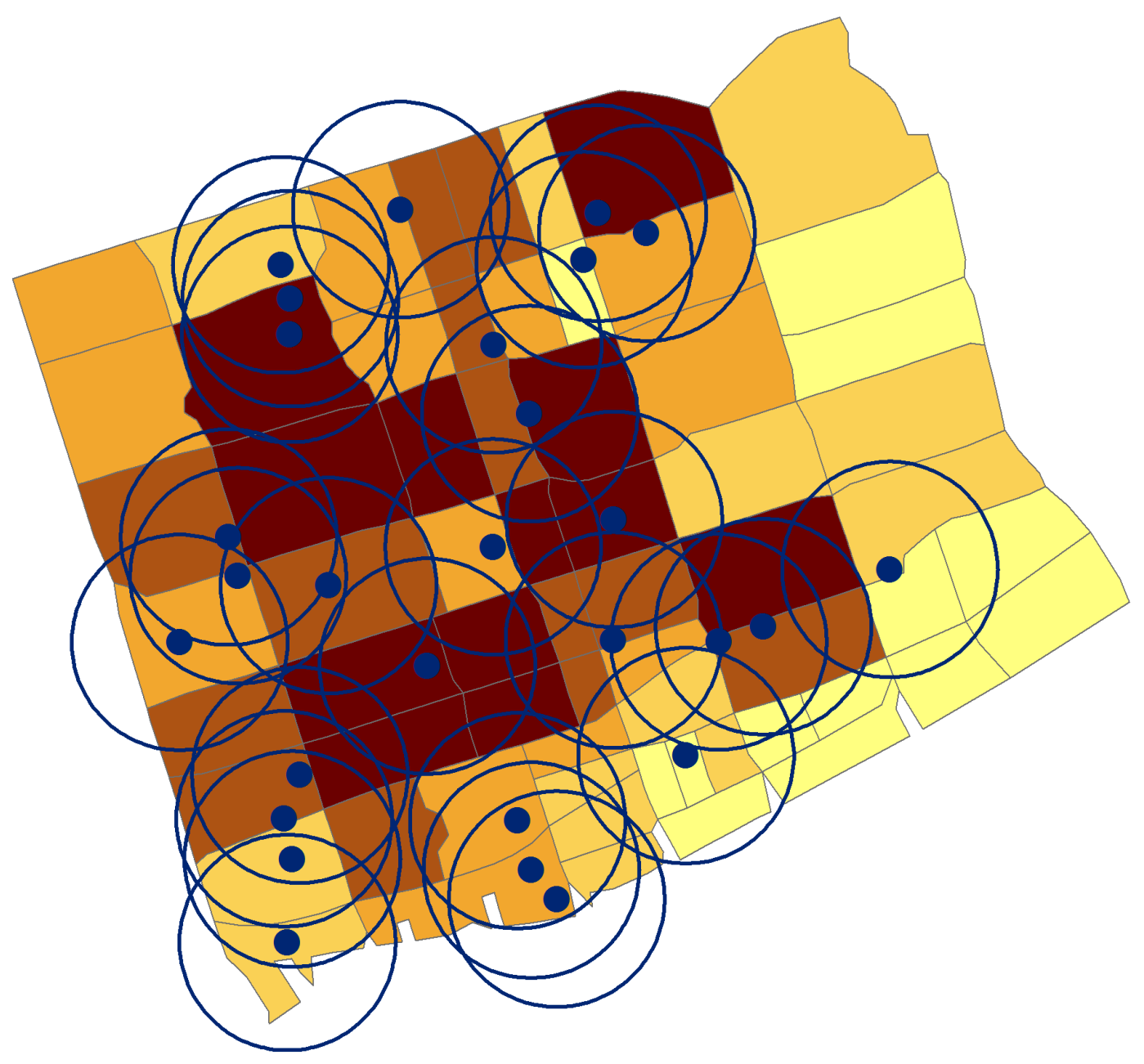

Figure A.13: Visualized result for SC-CP $(\mathrm{R}=500 \mathrm{~m}$, alpha $=90 \%$, beta $=1$, omega $=33 \%$, theta $=3$ ) 


\section{Bibliography}

Alexandris, G. and Giannikos, I. (2010). A new model for maximal coverage exploiting GIS capabilities. European Journal of Operational Research, 202(2), 328 - 338.

Buliung, R. N., Kanaroglou, P. S., and Maoh, H. (2005). GIS, Objects and Integrated Urban Models. In M. E. H. Lee-Gosselin and S. T. Doherty, editors, Integrated Land-Use and Transportation Models: Behavioural Foundations, pages 207-230. Elsevier.

Chen, N., Gardner, L. M., Duell, M., and Waller, S. T. (2014). Evaluating Location Alternatives for Electric Vehicle Re-charging Infrastructure Using a Distance Constrained Equilibrium Assignment Model. In Transportation Research Board 93rd Annual Meeting, number 14-1840.

Chen, T. D., Kockelman, K. M., Khan, M., et al. (2013). The electric vehicle charging station location problem: a parking-based assignment method for Seattle. In Transportation Research Board 92nd Annual Meeting, volume 340, pages 13-1254.

Community Energy Association (2013). Planning for Electric Vehicle Charging Infrastructure: A Toolkit. Report. 
M.Sc. Thesis - Xiaozhou Zhang McMaster - Computational Science and Engineering

Eberle, D. U. and von Helmolt, D. R. (2010). Sustainable transportation based on electric vehicle concepts: a brief overview. Energy Environ. Sci., 3, 689-699.

Frade, I., Ribeiro, A., Gonalves, G., and Antunes, A. (2011). Optimal Location of Charging Stations for Electric Vehicles in a Neighborhood in Lisbon, Portugal. Transportation Research Record: Journal of the Transportation Research Board, 2252, 91-98.

Ge, S., Feng, L., and Liu, H. (2011). The planning of electric vehicle charging station based on grid partition method. In 2011 International Conference on Electrical and Control Engineering (ICECE), pages 2726-2730.

Hanabusa, H. and Horiguchi, R. (2011). A Study of the Analytical Method for the Location Planning of Charging Stations for Electric Vehicles. In A. Knig, A. Dengel, K. Hinkelmann, K. Kise, R. Howlett, and L. Jain, editors, Knowledge-Based and Intelligent Information and Engineering Systems, volume 6883 of Lecture Notes in Computer Science, pages 596-605. Springer Berlin Heidelberg.

Hodgson, M. J. (1990). A Flow-Capturing Location-Allocation Model. Geographical Analysis, 22(3), 270-279.

Lam, A., Leung, Y.-W., and Chu, X. (2014). Electric Vehicle Charging Station Placement: Formulation, Complexity, and Solutions. Smart Grid, IEEE Transactions on, 5(6), 2846-2856.

Lam, W. H. K. and Lo, H. K. (2004). Traffic Assignment Methods. In D. A. Hensher, K. J. Button, K. E. Haynes, and P. R. Stopher, editors, Handbook of Transport Geography and Spatial Systems, pages 609-625. Elsevier, Oxford. 
M.Sc. Thesis - Xiaozhou Zhang McMaster - Computational Science and Engineering

Lee, Y.-G., Kim, H.-S., Kho, S.-Y., and Lee, C. (2014). UE-based Location Model of Rapid Charging Stations for EVs with Batteries that Have Different States-ofcharge. In Transportation Research Board 93rd Annual Meeting, number 14-3809.

Liu, J. (2012). Electric vehicle charging infrastructure assignment and power grid impacts assessment in Beijing. Energy Policy, 51, $544-557$. Renewable Energy in China.

Maoh, H. and Kanaroglou, P. (2009). A tool for evaluating urban sustainability via integrated transportation and land use simulation models. Environnement Urbain/Urban Environment, 3, 28-46.

McMaster Institute for Transportation and Logistics (2014). Estimating Vehicular Emissions for the Toronto and Hamilton Census Metropolitan Areas. Report.

Meyer, M. and Miller, E. (1984). Urban transportation planning: A decision-oriented approach. McGraw Hill, New York.

Miller, H. J. (1996). GIS and geometric representation in facility location problems. International Journal of Geographical Information Systems, 10(7), 791-816.

Murray, A. T. (2005). Geography in Coverage Modeling: Exploiting Spatial Structure to Address Complementary Partial Service of Areas. Annals of the Association of American Geographers, 95(4), 761-772.

National Renewable Energy Laboratory (2014). California Statewide Plug-in Electric Vehicle Infrastructure Assessment. Report.

Rashidi, T. H., Kanaroglou, P., Toop, E., Maoh, H., and Liu, X. (2015). Emissions 
M.Sc. Thesis - Xiaozhou Zhang McMaster - Computational Science and Engineering

and built form an analysis of six Canadian cities. Transportation Letters, $\mathbf{7}(2)$, $80-91$.

Sheffi, Y. (1995). Urban Transportation Networks: Equilibrium Analysis with Mathematical Programming Methods. Prentice-Hall.

U.S. Department of Energy (2014). Compare All-Electric Vehicles Side-by-Side.

Wong, D. (2009). The modifiable areal unit problem (MAUP). In A. S. Fotheringham and P. A. Rogerson, editors, The SAGE Handbook of Spatial Analysis, pages 105124. Sage Publications, Oxford.

Xi, X., Sioshansi, R., and Marano, V. (2013). Simulation-optimization model for location of a public electric vehicle charging infrastructure. Transportation Research Part D: Transport and Environment, 22, $60-69$. 